\title{
Increasing the forecasting fidelity of current collection system operating capability by means of contact pressure simulation modelling
}

\author{
Anton Golubkov ${ }^{1}$, Gleb Ermachkov ${ }^{1}$, Aleksandr Smerdin $^{1,},{ }^{*}$ Oleg Sidorov ${ }^{1}$, and Victor \\ Philippov $^{1}$ \\ ${ }^{1}$ Omsk State Transport University, Marx av., 644046, 35, Omsk, Russia
}

\begin{abstract}
Current collection quality is one of the limiting factors when increasing trains movement speed in the rail sector. With the movement speed growth, the impact forces on the current collector from the rolling stock and the aerodynamic influence increase, which leads to the spread in the contact pressure values, separation of the current collector head from the contact wire, contact arcing and excessive wear of the contact elements. The upcoming trend in resolving this issue is the use of the automatic control systems providing stabilization of the contact pressure value. The present paper considers the features of the contemporary automatic control systems of the current collector's pressure; their major disadvantages have been stated. A scheme of current collector pressure automatic control has been proposed, distinguished by a proactive influence on undesirable effects. A mathematical model of contact strips wearing has been presented, obtained in accordance with the provisions of the central composition rotatable design program. The analysis of the obtained dependencies has been carried out. The procedures for determining the optimal current collector pressure on the contact wire and the pressure control principle in the pneumatic drive have been described.
\end{abstract}

\section{Introduction}

The main directions of technological advancement of electric railway systems are increasing speed and capacity of passengers traffic and cargo transportation.

The one of the biggest difficulties in high speed railroads operation is solving the problem of safe and reliable power transfer to the trains. Current collector is a electromechanical device mounted on a roof of an electric train and designated to transfer power to train through contact with overhead line (catenary).

The power collection quality and the wear of both pantograph and catenary highly depend on pantograph dynamic behavior. Both current collector and overhead line don't have reserve and single failure in one of them may cause damage in the other one. Damage of current collector or catenary will cause trains delays and significant financial losses.

"Corresponding author: alexandr.smerdin@omgups.com 
Currently, one of the principal directions of the railway transport development is highspeed running [1]. Effective current collectors operation, specifically when increasing the movement speed, involves the expenses decrease on their maintenance and repair. The increase in movement speed is connected with the growth of the load on the electric locomotive resulting in the rise in failures of its parts and assemblies. In such a case, the repair interval of the entire electric locomotive should coincide with the current collectors repair interval.

\section{Materials and methods}

\subsection{Failure analysis of electric locomotives during operation}

According to data collected in service locomotive depots in trans-urals and perm in 20172018 [2], a quarter of all electric motive power failures are caused by current collectors (Fig. 1 (a)). In this event, the least fault-tolerant elements of the current collector are contact strips (Fig. 1 (b)), which clearly indicate the consequences of poor-quality current collection, resulted in the excessive wear of the contact surface [3]. Besides, the unevenly worn contact strip increases the pressure spread thereby deteriorating the quality of the current collection and enhancing the likelihood of other current collector's unit's failure.

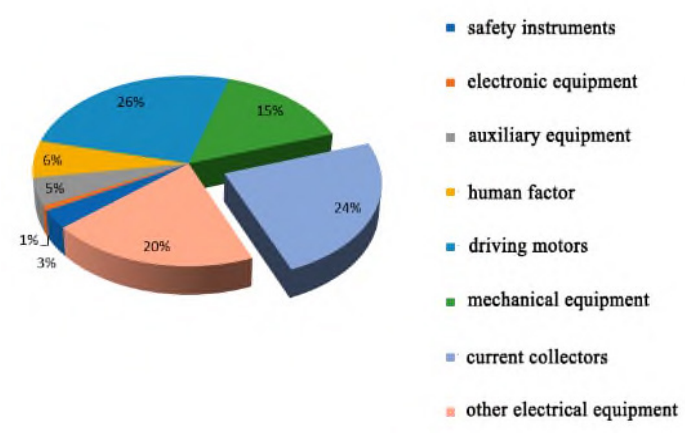

(a)
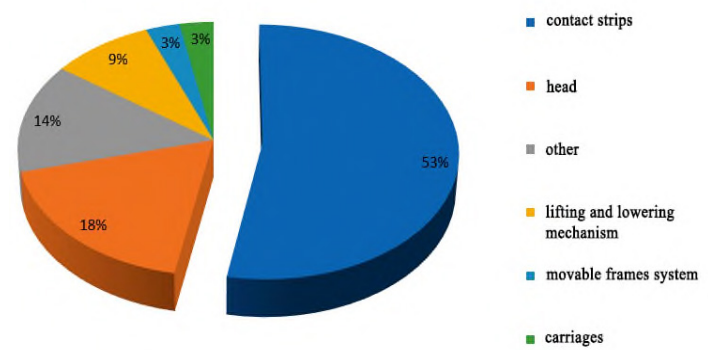

(b)

Fig. 1. Electric locomotive (a) and current collector (b) failure pattern.

\subsection{Background of increasing efficiency of exploitation of pantographs}

One of the ways to ensure the quality and reliability of current collection is to control the pressure in the pneumatic drive of the current collector. 
During the service checks due to increase in the movement speed, climatic conditions worsening or poor quality adjustment of the catenary, an increased spread of pressure takes place which leads to sparking and arcing.

At present, there is a considerable number of development works devoted to the pressure control accumulated over several decades, however, the implementation of these development works is virtually absent due to the high cost, complexity of installation and maintenance.

The use of the pressure control system in comparison with a system without control significantly reduces the number of sparks between the head and the contact wire as well as reduces the contact elements wear. However, the system's disadvantage is its reaction to the undesirable event after their occurrence. The result of this reaction may have a negative effect, the example of which can be observed in the section of 600-1200 m (Fig. 2), where the increase in pressure caused the excessive wear of the elements.
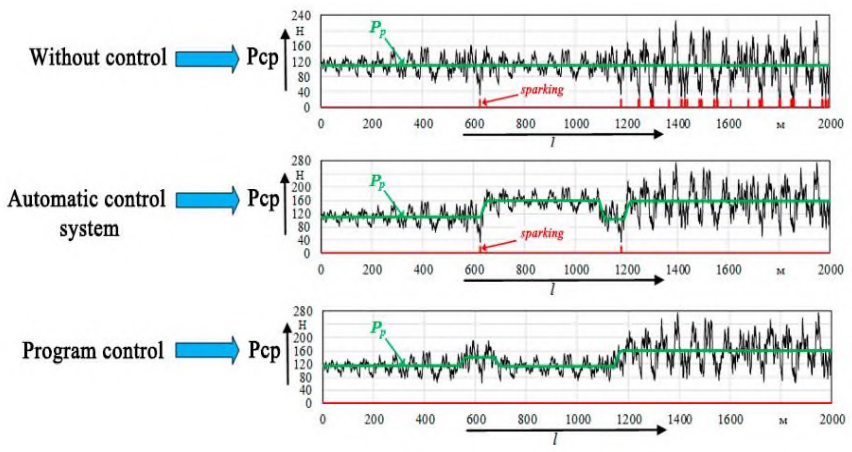

Fig. 2. Comparison of the current collector pressure control systems.

The proposed solution involves pressure program control in the pneumatic drive of the current collector with a proactive effect. This system, before the current collection quality starts to grow worse, begins to respond in advance, achieving optimal contact pressure throughout the length of the sector under the conditions of the minimal wear of the contact strips.

\subsection{Determination of wear parameters of current-removing elements in laboratory conditions}

To determine the optimal average contact pressure, the laboratory testing of the strips wear was carried out using a ring stand to simulate the interaction of current collectors with contact suspensions. The stand provides a load current of up to $4000 \mathrm{~A}$ with drop in voltage up to $15 \mathrm{~V}$ [4].

The tests were carried out with a change in three main factors (contact pressure, traction current and movement speed). The force of contact pressure is formed by the following components:

$$
P=P_{\text {stat }}-P_{\text {aero }}-P_{\text {dyn }}
$$

where $P_{\text {stat }}$ - force generated by the current collector drive during the rolling stock standing time;

$P_{\text {aero }}$ - increase in the contact pressure force as a result of aerodynamic influence on the current collector; 
$P_{d y n}$ - the contact pressure force component resulting from the interaction of the current collector and contact suspension.

Taking into account the fact that during the tests there was no aerodynamic influence on the current collector and the number of separations of the current collector from the contact wire was insignificant, the following assumption was made:

$$
P=P_{\text {stat }}
$$

Based on a series of studies [5-8], it was found that the dependence $j=f(P, I, \mathrm{v})$ of the collection strips wear is non-linear. Therefore, it is advisable to conduct a study of this process in accordance with the provisions of the second-order central composition rotatable design program [9].

The contact strips wear is calculated by piecewise-polynomial approximation of the formed wear surface according to the procedure [10].

The procedure for the wear study includes the following steps:

- A study of the contact strips wear from contact pressure and movement speed (current load is absent);

- A study of the contact strips wear depending on the contact pressure, movement speed and current load. The values of the maximum permissible current are specified according to the conditions of thermal overload of the contact elements (based on the values specified in the technical conditions) as well as when registering the increased sparking (arcing).

At each stage, a range of rational values of the studied factors and the optimal amount of experiments are determined.

The primary data for the experiment is presented in Table 1. The levels and facts variability intervals are given in Table 2.

Table 1. Primary data for the experiment.

\begin{tabular}{|c|c|}
\hline Contact strip type & RH85M8* \\
\hline Contact plate material & $\mathrm{M} 1$ \\
\hline Dust content & $10 \mathrm{mg} / \mathrm{m} 3$ \\
\hline Humidity & $20 \%$ \\
\hline Air temperature & $22^{\circ} \mathrm{C}$ \\
\hline Contact wires zigzag & $\pm 300 \mathrm{~mm}$ \\
\hline
\end{tabular}

Table 2. Levels and facts variability intervals.

\begin{tabular}{|c|c|c|c|c|c|c|}
\hline \multirow{2}{*}{ Factor } & \multirow{2}{*}{ Code designation } & \multicolumn{5}{|c|}{ Real factors levels corresponding to the coded } \\
\cline { 3 - 7 } & & $+1,682$ & +1 & 0 & -1 & $-1,682$ \\
\hline $\mathrm{P}, \mathrm{H}$ & $\mathrm{x}_{1}$ & 200 & 160 & 120 & 80 & 40 \\
\hline $\mathrm{I}, \mathrm{A}$ & $\mathrm{x}_{2}$ & 900 & 675 & 450 & 225 & 0 \\
\hline $\mathrm{v}, \mathrm{km} / \mathrm{h}$ & $\mathrm{x}_{3}$ & 250 & 200 & 150 & 100 & 50 \\
\hline
\end{tabular}

Based on the levels and facts variability intervals, an experiment matrix was made. The second-order central composition rotatable design for three factors consists of a complete factorial design of type 23 (experiments 1-8), six experiments at "star" points (experiments 9-14) and six experiments in the center point of design (experiments 15-20). The number of experiments in the center point of design determines the pattern of distribution of the received information on the response surface and is selected in accordance with the uniform-rotatability conditions. Uniform-rotatable design is possible if some constant $\lambda$ does not exceed unity (shade less than it):

$$
\lambda=\frac{k \cdot\left(n_{c}+n_{0}\right)}{(k+2) \cdot n_{c}}
$$


where $k$ - number of factors;

$n_{0}$ - number of experiments in the center point of design (number of zero points);

$n_{c}$ - difference between the total number of experiments $N$ and experiments in the center point of design.

$$
n_{c}=N-n_{0} .
$$

The calculation showed that the selected number of experiments in the center point of design corresponds to uniform-rotatability, since $\lambda=0.857$.

The resulting three-factor dependence of the current-collecting strips wear can be represented as a family of mathematical matrices (Table 3 ).

Table 3. Three-factor dependence of the current-collecting strips wear as a family of mathematical matrices.

\begin{tabular}{|c|c|c|c|c|c|c|c|c|c|}
\hline$v$ & \multirow{2}{*}{\multicolumn{4}{|c|}{$\frac{v_{1}}{P_{1}}$}} & $\gamma$ & \multirow{2}{*}{\multicolumn{4}{|c|}{$v_{i}$}} \\
\hline$\gamma$ & & & & & $\gamma$ & & & & \\
\hline$I_{1}$ & $P_{11}$ & $P_{21}$ & $\ldots$ & $P_{n 1}$ & $I_{i}$ & $P_{1 i}$ & $P_{2 i}$ & $\ldots$ & $P_{n i}$ \\
\hline$I_{11}$ & $j_{111}$ & $j_{211}$ & $\ldots$ & $j_{n 11}$ & $I_{1 i}$ & $j_{11 i}$ & $j_{21 i}$ & $\ldots$ & $j_{n 1 i}$ \\
\hline$I_{21}$ & $j_{121}$ & $j_{221}$ & $\ldots$ & $j_{n 21}$ & $I_{2 i}$ & $j_{12 i}$ & $j_{22 i}$ & $\ldots$ & $j_{n 2 i}$ \\
\hline$\ldots$ & $\ldots$ & $\ldots$ & $\ldots$ & $\ldots$ & $\ldots$ & $\ldots$ & $\ldots$ & $\ldots$ &. \\
\hline$I_{w 1}$ & $j_{1 w 1}$ & $j_{2 w 1}$ & $\ldots$ & $j_{n 1}$ & $I_{w i}$ & $\bar{j}_{1 w i}$ & $j_{2 w i}$ & $\ldots$ & $j_{n w i}$ \\
\hline
\end{tabular}

Following the results of experiments performed in accordance with the experimental design, the beta coefficients were estimated:

$$
\begin{aligned}
& j=b_{0}+b_{1} \cdot x_{1}+b_{2} \cdot x_{2}+b_{3} \cdot x_{3}+ \\
& +b_{12} \cdot x_{1} \cdot x_{2}+b_{13} \cdot x_{1} \cdot x_{3}+ \\
& +b_{23} \cdot x_{2} \cdot x_{3}+b_{11} \cdot x_{1}^{2}+b_{22} \cdot x_{2}^{2}+b_{33} \cdot x_{3}^{2} .
\end{aligned}
$$

The equation coefficients (5) were determined based on the number of experiments in the center point of design, the number of factors and the total number of experiments [9]. For $\mathrm{k}=3$ formulas for calculating the equation coefficients:

$$
\begin{aligned}
& b_{0}=0.16666 \cdot \sum_{q=1}^{20} j_{q}-0.05694 \cdot \sum_{i=1}^{3} \sum_{q=1}^{20} j_{i q}^{2} y_{q}, \\
& b_{i}=0.07322 \cdot \sum_{q=1}^{20} x_{i q} \cdot j_{q}, \\
& b_{i l}=0.125 \cdot \sum_{q=1}^{20} x_{i q} \cdot x_{l q} \cdot j_{q}, \\
& b_{i i}=0.06254 \cdot \sum_{q=1}^{20} x_{i q}^{2} \cdot j_{q}+0.00695 \times \\
& \times \sum_{i=1}^{3} \sum_{q=1}^{20} x_{i q}^{2} \cdot j_{q}-0.05694 \cdot \sum_{q=1}^{20} j_{q} .
\end{aligned}
$$


Sums included in the formulas for calculating the equation coefficients:

$$
\begin{aligned}
& \sum_{q=1}^{20} j_{q}=3.09299 \\
& \sum_{q=1}^{20} x_{1 q} j_{q}=-0.04312 \\
& \sum_{q=1}^{20} x_{2 q} j_{q}=0.72835 \\
& \sum_{q=1}^{20} x_{3 q} \cdot j_{q}=0.968144 \\
& \sum_{q=1}^{20} x_{1 q} \cdot x_{2 q} \cdot j_{q}=-0.1234 \\
& \sum_{q=1}^{20} x_{1 q} \cdot x_{3 q} \cdot j_{q}=0.1146 \\
& \sum_{q=1}^{20} x_{2 q} \cdot x_{3 q} \cdot j_{q}=0.1054 \\
& \sum_{q=1}^{20} x_{1 q}^{2} \cdot j_{q}=2.974539 \\
& \sum_{q=1}^{20} x_{2 q}^{2} \cdot j_{q}=1.999849 \\
& \sum_{q=1}^{20} x_{3 q}^{2} \cdot j_{q}=2.330677 \\
& \sum_{q=1}^{20} x_{i q}^{2} \cdot j_{q}=7.305064
\end{aligned}
$$

Using formulas (6)-(9), the beta coefficients were determined. As a result of the rotatable design of the second order use, after eliminating of the insignificant coefficients [9], the following regression equation was obtained in the encoded form:

$$
\begin{aligned}
& j=0.099527-0.00316 \cdot x_{1}+0.05333 \cdot x_{2} \\
& +0.070888 \cdot x_{3}-0.01543 \cdot x_{1} \cdot x_{2}+ \\
& +0.014325 \cdot x_{1} \cdot x_{3}+0.013175 \cdot x_{2} \cdot x_{3}+ \\
& +0.060683 \cdot x_{1}^{2}-0.00027 \cdot x_{2}^{2}+0.020416 \cdot x_{3}^{2} .
\end{aligned}
$$

Testing the hypothesis of the model adequacy by the F-criterion [9] demonstrated that the model is adequate on 5\% significance level, since the calculated value of the F-criterion $(\mathrm{Fp}=3.72)$ is less than the tabular $(\mathrm{FT}=4.57)$. The values of $\mathrm{j}$ calculated by Equation $(10)$ differ from the experimental by values that do not exceed the experimental error.

Moving from the coded $\mathrm{x} 1, \mathrm{x} 2, \mathrm{x} 3$ factors values with account taken of the variation intervals to natural $\mathrm{P}, \mathrm{I}, \mathrm{v}$, the dependence of the contact strips wear of electric motive power's current collector on the operating parameters was obtained: 


$$
\begin{aligned}
& j=633895 \cdot 10^{-6}+8 \cdot 10^{-6} \cdot v^{2}+271 \cdot 10^{-6} \cdot I+ \\
& +3.79 \cdot 10^{-5} \cdot P^{2}-5.41 \cdot 10^{9} \cdot I^{2}- \\
& -2418 \cdot 10^{-6} \cdot v-9484 \cdot 10^{-6} \cdot P+ \\
& +1.17 \cdot 10^{-6} \cdot I \cdot v+7.16 \cdot 10^{-6} \cdot P \cdot v- \\
& -1.71 \cdot 10^{-6} \cdot P \cdot I .
\end{aligned}
$$

For the purpose of increasing the experimental results reliability, the experiments were repeated. For the repeated experiment, the matrices were implemented with a changed order of experiments (combinations of factors did not change).

According to the data obtained, the characteristics of contact strips wear are constructed, which allow to determine the best contact pressure of the current collector on the contact wire, providing minimal electrical and mechanical wear for the studied friction pair.

Subsequent to the results of the experiment, the surface of the contact strips wear rate from pressure value and current flow strength in contact (at a speed of $150 \mathrm{~km} / \mathrm{h}$ ), as well as the surface of the contact strips wear rate from pressure value and movement speed at a constant current load of 1500 A were built.

Analysis of the results allows for the conclusion that the optimal range of contact pressure with an increase in current load and movement speed, shifts to the right.

To calculate the life cycle of the current collector's contact plates along with the wear dependences, it is necessary to have a graph of the pressure change in contact, as well as graphs of the movement speed and the current consumption in the reviewed section. Graphs of the movement speed and current consumption in the reviewed section can be obtained from the electric motive power schedule graph. The most convenient way to obtain a data set $\mathrm{P}$ and to develop operation algorithms is mathematical simulation modelling based on the INS use.

Whereby the obtained values are statistically reliable and the signal's spectral power density corresponds to the real record $\mathrm{P}$ on the active line [11-14]. The model allows to vary over a wide range such parameters as electric motive power speed, elasticity and spatial arrangement of contact wires, which remarkably accelerated the testing process.

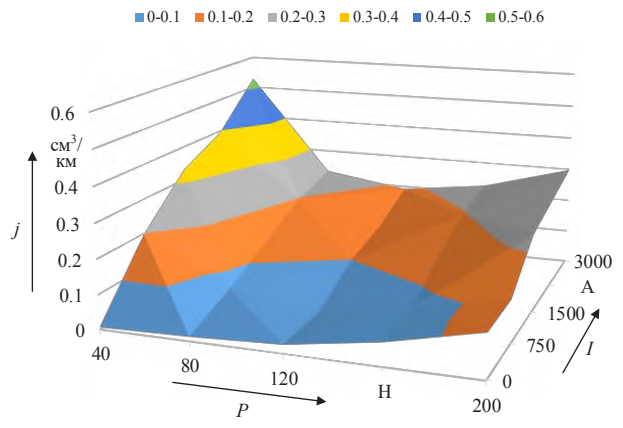

a

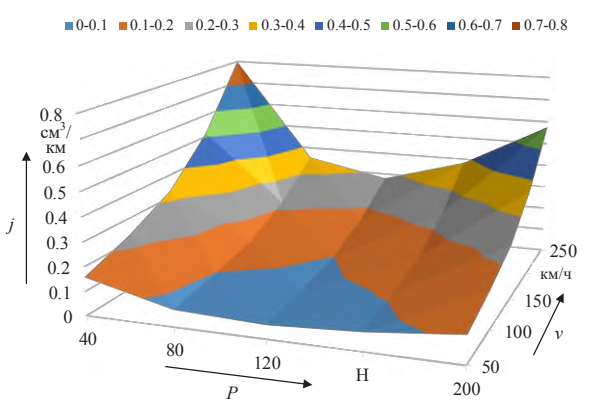

b

Fig. 3. Surface of the contact strips wear rate a) from pressure value and current flow strength in contact (at a speed of $150 \mathrm{~km} / \mathrm{h}$ ); b) from pressure value and movement speed (at a current load of $1500 \mathrm{~A})$.

\subsection{Development of a device for programmatic control pressure on the contact wires}

The optimal P values for each section are determined by iterational method, by calculating the specific wear at various values of the electric motive power speed, current and average contact pressure. Each obtained $\mathrm{P}$ value corresponds to a pressure value in the pneumatic 
drive $\rho$ taking into account the lengths of the control sectors L. Determination of $L$ is conducted with the consideration of the movement speed and the device response time.

The received data is stored in a multidimensional table for the reference speed values (Fig. 4). For intermediate speed values, the optimal pressure $\rho$ is determined by interpolating the previously obtained data. The control cards recorded in the memory of the control device are selected in accordance with the readings of the position, current, and electric motive power speed sensors. The length of sections with constant pressure L depends on the system response time, the movement speed and the number of control steps.

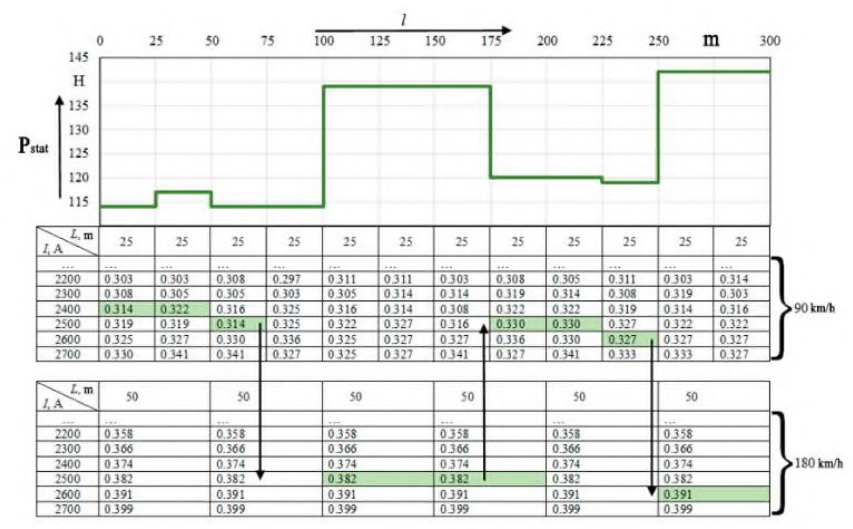

Fig. 4. Optimum pressure selection procedure ( $\rho, \mathrm{MPa})$ from the automated control system memory unit at various electric motive power speed values.

To implement the proposed approach to controlling the current collector pressure, a technical solution was developed, its operating principle is based on the control of electropneumatic valves regulating the pressure in the pneumatic system by means of the control unit, which in its turn receives information from a set of sensors located on the electric motive power (Fig. 5).

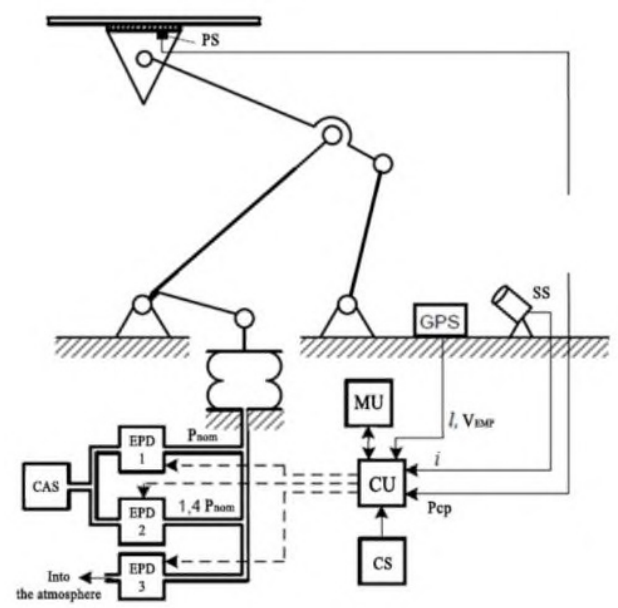

Fig. 5. Pressure program control: $\mathrm{PS}$ - pressure sensor; $\mathrm{SS}$ - sparking sensor; CU - control unit; $\mathrm{MU}$ - memory unit; CS - current sensor.

The model of the present technical solution was made and is being tested in the laboratory "Catenary, power lines and current transfer design" at Omsk State Transport University as a control device of the current collector Siemens SSS-87 for Russian 
Railways.

The program control system takes priority control action based on the readings from the position sensor and the known values of current and speed from the electric motive power movement schedule, lined up to the location data (Fig. 6). When deviating from the movement schedule, the control system takes account of the current readings from the current and speed sensors.

If an unforeseen situation takes place, such as ice conditions, which cause abnormal operation, automatic control is carried out upon the adverse events occurrence, based on readings from sparking and pressure sensors.

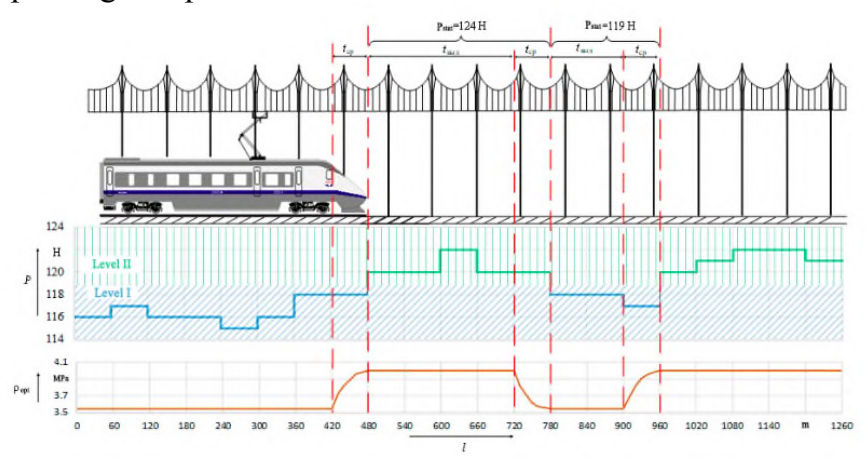

Fig. 6. Operating principle of the automatic control system at the two-step pressure control in the pneumatic drive $t_{d r}$ - device response time; $t_{d}$ - delay time.

The suggested device exercises control using the two levels of regulation and for which reason can already be used on the following electric locomotives: EP20 "Olimp", EVS2 "Sapsan" and ED4M "Lastochka".

\section{Conclusion}

Thus, according to the results of the study, it is possible to make the following conclusions:

- Program pressure control in the pneumatic system of the current collector allows to advance the efficiency of its operation by increasing the life cycle;

- To justify the optimal values of the average contact pressure on the existing and planned lines, it is expediently to carry out preliminary wear tests according to the secondorder central composition rotatable design program;

- Available hardware in the pneumatic system of current collectors on the advanced electric motive power can be used to create the proposed hardware-software system for the pressure control.

\section{References}

1. Updated program of high-speed railway communication on the territory of the Russian Federation.

2. A. P. Buinosov, K. G. Shumakov, "Method for estimating the technical condition of electric truck receiver (Periodical style)" Scientific and Technical Bulletin of the Volga Region, 9, 15 - 18 (2019)

3. A. N. Smerdin, "Improvement the current collection system of main electric railways under conditions of high-speed and heavy haul movement (Doctor of Technical Science thesis work: 05.22.07)”, 600 (2019) 
4. O. A. Sidorov, V. M. Pavlov, "The study of the current collector current load capacity of the main electric motive power (Periodical style)" Railway research institute bulletin, 4, $19-24$ (2015)

5. O. A. Sidorov, S. A. Stupakov, V. M. Philippov, "The simulation of the current collection devices' contact pairs wear of monorail electric vehicles (Periodical style)" Journal of the Transsib Railway Studies, 3(7), 43 - 52 (2011)

6. R. G. Bayer, "Prediction of Wear in a Sliding System (Periodical style)" Wear, 11, 319-332 (1968)

7. A. Collina, S. Melzi, "Effect of contact strip-contact wire interaction on current transfer at high sliding speed in the mid-high frequency range (Periodical style)", 5th International Conference on Tribology AITC-AIT 2006, (2006)

8. G. X. Chen, H. J. Yang, W. H. Zhang, X. Wang, S.D. Zhang, and Z.R. Zhou, "Experimental study on arc ablation occurring in a contact strip rubbing against a contact wire electrical current”, Tribology Int., 61, 88-94 (2013)

9. A. A. Spiridonov, "Planning an experiment when studying technological processes", Mechanical Engineering, 184 (1981)

10. A. N. Smerdin, A. E. Chepurko, "Analysis of the environmental load from the electrified transport current collection system”, Ural Transport, 1 (56), 69 - 74 (2018)

11. G. R. Ermachkov, A. S. Golubkov, et al., "Application of neural networks at simulation of a current collection system on electric railways" Journal of the Transsib Railway Studies, 1(33), 69 - 79 (2018)

12. S. Bruni, J. Ambrosio, A. Carnicero, Y. H. Cho, L. Finner, M. Ikeda and W. Zhang, "The Results of the Pantograph-Catenary Interaction Benchmark" Veh. Syst. Dyn., 53(3), 412-435 (2015)

13. A. V. Paranin, A. V. Efimov, D. V. Efimov, "Modelling of the clean contact between the contact wires and collector strip with static finite element methods" Journal of the Transsib Railway Studies, 1(17), 57 - 67 (2014)

14. L. Zhigang, Y. Song, Y. Han, H. Wang, J. Zhang, Z. Han, “Advances of research on high-speed railway catenary” Journal of Modern Transportation, 26(1), 1-23 (2018) 